\section{Desigualdades socioeconómicas en la utilización de servicios de salud bucal: estudio en escolares mexicanos de 6 a 12 años de edad}

\author{
Socioeconomic inequalities in the use of dental \\ services: a study of Mexican schoolchildren \\ aged 6 to 12 years
}

\footnotetext{
${ }^{1}$ Instituto de Ciencias de la Salud, Universidad Autónoma del Estado de Hidalgo, Pachuca, México. 2 Unidad de Medicina Familiar, Instituto de Seguridad y Servicios Sociales de los Trabajadores del Estado, Navolato, México. ${ }^{3}$ Facultad de Odontología, Universidad Autónoma de Sinaloa, Culiacán, México. 4 Facultad de Odontología, Universidad Autónoma de Campeche, Campeche, México.

5 Delegación del Instituto de Seguridad y Servicios Sociales de los Trabajadores del Estado, Culiacán, México. 6 Facultad de Medicina, Universidad Autónoma de Sinaloa, Culiacán, México.

Correspondencia C. E. Medina-Solís Área Académica de Odontología, Instituto de Ciencias de la Salud, Universidad Autónoma del Estado de Hidalgo. Privada de Altillo s/ $n$ entre $A v$. Central y Pedro Moreno, Colonia San José, C.P. 24040 , Campeche, Campeche, México.

cemedinas@yahoo.com
}

\begin{abstract}
The aim of this cross-sectional study was to evaluate the association between socioeconomic indicators and use of dental services in a sample of 3,048 Mexican schoolchildren. The dependent variable "use of dental services" and independent variables were collected through a questionnaire addressed to mothers. To determine oral health needs, a clinical oral examination was performed. The adjusted associations were evaluated using polytomous logistic regression. Adjusted by gender, the categories associated with both preventive and curative services were age, greater frequency of brushing, earlier initiation of brushing, healthcare coverage, and better socioeconomic status. In addition, for preventive services only, use of services was associated with enrolment in private school, and for curative services only, family's possession of an automobile and having moderate to high oral health needs. The results suggest the existence of socioeconomic inequalities in the use of both preventive and curative dental services by Mexican children.
\end{abstract}

Dental Health Services; Health Services Research; Inequalities in Health; Child
Carlo Eduardo Medina-Solís 1

Juan José Villalobos-Rodelo 2,3 María de Lourdes Márquez-Corona 1 Ana Alicia Vallejos-Sánchez 4 Carlos López Portillo-Núñez 5,6 Alejandro José Casanova-Rosado 4

\section{Introducción}

Con base en diversos estudios, existe en la literatura científica y profesional un amplio consenso en cuanto al efecto negativo que tiene la magnitud y distribución de las desigualdades socioeconómicas (empleando diferentes indicadores de posición socioeconómica) sobre distintos aspectos relacionados a la salud. Algunos de estos son la morbilidad 1,2,3,4,5,6, la mortalidad 7,8,9, así como con la prestación de servicios de salud en la población 1,10,11,12. La dirección general que sigue esta relación es que a medida que desciende la posición socioeconómica, los indicadores de salud son peores. El caso de la salud bucal es similar. Empleando distintos indicadores de posición socioeconómica durante los últimos años en países latinoamericanos, como Brasil y México, se ha incrementado el número de estudios donde se demuestran las desigualdades socioeconómicas que existen en los indicadores de salud bucal 13 , $14,15,16,17,18,19,20,21$, así como en la cobertura y utilización de servicios odontológicos 22,23,24,25,26.

Una buena parte del nivel del estado de salud bucal en la comunidad puede ser atribuido a la prestación específica de servicios. En odontología comunitaria existe un interés creciente sobre conocer cuáles son los determinantes que provocan que las personas utilicen servicios de salud bucal, así como distinguir entre los usuarios que visitan al dentista para visitas de revisión de rutina, y aquellos que visitan al dentista por 
dolor, infección o tratamientos curativos 27. Esta información es crucial para poder diseñar programas o políticas que estimulen la utilización de servicios y disminuyan las brechas sociales que existen en cuanto al acceso en este tipo de servicios de salud 28,29,30. A pesar de la evidente importancia del tema, se han realizado pocos estudios para documentar la utilización de servicios de salud bucal en México 22,23,24,25 y menos aún si nos referimos al estudio diferenciando por tipo de servicio. Comúnmente, en la población general se ha visto que el ingreso, el área de residencia, el aseguramiento, la educación, y la ocupación son citados como predictores de la utilización de servicios de salud odontológicos preventivos y curativos, entre otros indicadores socioeconómi$\cos 22,23,24,25,29,31,32,33,34,35,36$.

Para estudiar el problema de la utilización de servicios de salud se han propuesto diferentes modelos teóricos 37 . El propuesto por Andersen \& Newman 38 integra a las variables independientes en tres grupos, que actúan influyendo sobre la conducta del individuo durante la búsqueda de atención de algún servicio de salud: (a) las variables predisponentes, que son características que condicionan una mayor probabilidad de utilizar los servicios, como las variables sociodemográficas, las creencias y actitudes sobre la enfermedad y algunos aspectos relacionados con la familia, como son su estructura y el número de integrantes; (b) las variables facilitadoras o capacitantes, que pueden impedir o facilitar el uso de los servicios, entre las que se encuentran el ingreso, los seguros médicos o dentales, la accesibilidad geográfica y aspectos relacionados con la organización misma de los servicios, incluyendo la relación médico-paciente; y finalmente, (c) las variables de necesidad, entendidas como un cambio en el estado de salud. Este cambio puede ser valorado de acuerdo al punto de vista del profesional (valoración técnica o normativa), o bien según la percepción del individuo enfermo y su familia (valoración subjetiva, o sentida/expresada). Este modelo teórico se ha utilizado en estudios sobre servicios odontológicos en diversos escenarios 22,23,24,35,39,40,41. Dentro de este modelo es posible estudiar al mismo tiempo la existencia de desigualdades en salud, ya que en él se incluyen variables socioeconómicas, donde si existiese desigualdad socioeconómica sería viable identificarla.

El objetivo de este estudio fue evaluar la asociación entre diversos indicadores socioeconómicos y la utilización de servicios de salud bucal, diferenciándolos por tipo de servicio utilizado en niños de seis a 12 años de edad en Navolato, Sinaloa, México.

\section{Material y métodos}

\section{Diseño, población y muestra de estudio}

Sinaloa se ubica en el Noroeste de México, limita al norte con los estados de Sonora y Chihuahua; al este con Durango y Nayarit; al sur con Nayarit y el Océano Pacífico; y al oeste con el Golfo de California y Sonora. El municipio de Navolato (donde se realizó el estudio) se encuentra en el centro del estado, con una densidad de población de 51-100 por km². El municipio cuenta con una población total de 135.681; la población de 6 a 14 años fue de 26.418. Ocupa el quinto lugar en población de los 18 municipios del estado de Sinaloa $(5,7 \%)$. La población derechohabiente es casi un $65 \%$. El índice de marginación a nivel municipal (IMM) es de -0,65194, lo que lo ubica en el grupo de municipios de México catalogados con bajo IMM, esto es, tiene baja inequidad socio-espacial (Consejo Nacional de Población; http://www.conapo.gob.mx/publicaciones/in dice2005.htm, accedido el 22/Ago/2007). La economía está sustentada principalmente en la producción de diversos productos agrícolas para la exportación y distribución nacional, otras actividades económicas son la industria azucarera la producción ganadera, pesquera, acuacultura, textil y explotación de sal. El Estado participa en el Programa Nacional de Fluoruración de la Sal, y de acuerdo a la Asociación Mexicana de la Industria Salinera se distribuye dos tipos de sal: sal yodada y sal yodada-fluorurada. Solamente la capital del estado (Culiacán) se encuentra en el listado de localidades en donde no se debe consumir sal fluorurada total o parcialmente (Asociación Mexicana de la Industria Salinera; http:// www.amisac.org.mx/page31.html, accedido el 22/Ago/2007)/(Comisión Federal para la Protección contra Riesgos Sanitarios; http://www. cofepris.gob.mx/inf/sal/listado_sal_yodada. htm, accedido el 22/Ago/2007).

Este informe forma parte de un proyecto integral sobre salud bucal en escolares de Navolato, Sinaloa 42,43. Su realización cumplió con las especificaciones de protección a los participantes en estudios y se adhirió a las reglamentaciones éticas y de investigación en vigor del Instituto $\mathrm{Na}$ cional de Salud Pública (INSP) en México. Se realizó un estudio transversal en niños de 6 a 12 años de edad, asistentes a las 18 escuelas primarias de la ciudad de Navolato. Asimismo, parte de la metodología ha sido publicada previamente 42,43 Brevemente, la población inicial consistió de 3.547 niños. En un principio, se habló con los representantes del sector salud y educativo de la ciudad; posteriormente se invitó a las madres/ tutores de los niños a participar en el estudio. Del 
total de niños, originalmente se obtuvo una participación de 75,4\% ( $\mathrm{n}=2.674)$. Tras un segundo recordatorio, se obtuvo una participación final de $87 \%$ ( $n=3.086$ ). Los niños fueron excluidos del estudio $(n=38)$ por alguna de las siguientes razones: (a) tenían menos de seis años y más de 12 años de edad, (b) tenían alguna enfermedad que afectaba la cavidad bucal, (c) rehusaron participar en el examen bucal, y (d) tuvieron aparatología ortodóntica fija. La muestra final fue de $3.048(85,9 \%)$ escolares.

\section{Variables del estudio y recolección de los datos}

La variable dependiente fue la utilización de servicios de salud bucal por el niño en los doce meses previos al estudio. Para su construcción y categorización se preguntó a las madres/tutores si los niños habían recibido algún tipo de atención dental, en cuyo caso se preguntó sobre el tipo de atención recibida. Para recolectar las variables sociodemográficas y socioeconómicas, se empleó un cuestionario dirigido a las madres/ tutores de los niños, posteriormente a la firma de la carta de consentimiento informado. Las variables independientes incluidas en el estudio fueron edad, sexo, edad de inicio de cepillado dental y su frecuencia, edad de ambos padres, y una serie de indicadores socioeconómicas como seguro de salud (incluye médico y dental), tipo de escuela, tamaño de la familia, posesión de automóvil en el hogar, y nivel socioeconómico. Para determinar una variable proxy a las necesidades de salud bucal, todos los sujetos fueron examinados clínicamente por uno de tres examinadores previamente capacitados y estandarizados (kappa > 0,85). El examen bucal consistió en la detección de la experiencia de caries (caries, obturaciones y extracciones), incluyéndose no sólo las lesiones obviamente cavitadas ${ }^{44}$, sino también las lesiones $\mathrm{d}_{1}$ del criterio de Pitts 45 . Las necesidades de salud bucal se establecieron con base en la severidad de la caries dental en ambas denticiones, siguiendo una estrategia similar a la empleada en otros estudios 22,23,24. Esta variable se codificó como necesidades de salud bajas (de 0 a 3), moderadas (de 4 a 6), altas (de 7 a 9), y muy altas (mayor de 9).

\section{Análisis estadísticos}

Primeramente, se utilizó el análisis de componentes principales (correlación policórica) 46, para conformar la variable nivel socioeconómico. Así, el nivel socioeconómico fue determinado utilizando la escolaridad y ocupación de ambos padres usando esta metodología, los cuatro factores socioeconómicos correlacionados entre sí fueron reducidos a un componente principal. Con el primer componente se logró explicar el 58,7\% de la variabilidad del nivel socioeconómico.

A continuación, se procedió con el análisis univariado; éste consistió en el cálculo de medidas de tendencia central y de dispersión para las variables continuas y porcentajes para las variables categóricas. En el análisis bivariado y multivariado se empleó el modelo de regresión logística multinomial. Dada la naturaleza de la variable dependiente, se analizó el tipo de servicio utilizado en el último año, categorizando la variable de respuesta como (0) no utilización de servicios odontológicos, (1) utilización de servicios odontológicos curativos, y (2) utilización de servicios odontológicos preventivos. Para la construcción del modelo final se tomaron en cuenta las variables que tuvieron en el análisis bivariado una significancia estadística $\mathrm{p}<0,20$. En el análisis se probó, si durante cada año de aumento para la variable independiente continua edad, el incremento en el logit de la variable de respuesta era constante (Box-Tidwell test). Se realizó la prueba de factor de inflación de la varianza (VIF) con el fin de analizar, y en su caso, evitar la multicolinealidad entre las variables independientes. Se probaron interacciones, pero ninguna resultó ser significativa a p $<0,1547,48$. Tanto en el análisis bivariado, como en el multivariado, los errores estándar fueron ajustados por la correlación que existe entre los niños del mismo colegio (cluster intra-escuela) y relativamente distinto entre los clusters 49 . Todos los análisis se realizaron en Stata 8.2 (Stata Corp., College Station, Estados Unidos).

\section{Resultados}

La Tabla 1 presenta la distribución de los indicadores socioeconómicos y de las características que describen la población estudiada. El promedio de edad fue $8,81 \pm 1,79$ años; la población incluyó 1.456 niños $(47,8 \%)$ y 1.592 niñas $(52,2 \%)$. Al menos la mitad de los niños $(56,3 \%)$ se cepillaban los dientes por lo menos una vez al día. En cuanto a la distribución de las variables socioeconómicas, el mayor porcentaje $(61,5 \%)$ de los niños tenía otros dos o más hermanos, sólo $15,5 \%$ asistía a escuelas privadas, $16,6 \%$ tenía un seguro privado de salud, y en 53,9\% tenían un automóvil. Por otro lado, $17,8 \%$ de los niños tuvieron necesidades bajas de salud, mientras que $28,8 \%$ presentaron muy altas necesidades de salud bucal.

La mayoría de los niños utilizó servicios curativos $(45,2)$, mientras que sólo $20,5 \%$ utilizó 
Tabla 1

Distribución de las características sociodemográficas y socioeconómicas de los niños incluidos en el estudio.

\begin{tabular}{|c|c|c|}
\hline Variables & Media \pm desviación estándar & Límites \\
\hline Edad del niño & $8,81 \pm 1,79$ & $6-12$ \\
\hline Edad del padre & $37,71 \pm 6,54$ & $20-68$ \\
\hline \multirow[t]{2}{*}{ Edad de la madre } & $34,64 \pm 5,72$ & $20-67$ \\
\hline & $\mathrm{N}$ & $\%$ \\
\hline \multicolumn{3}{|l|}{ Sexo } \\
\hline Niños & 1.456 & 47,8 \\
\hline Niñas & 1.592 & 52,2 \\
\hline \multicolumn{3}{|c|}{ Frecuencia de cepillado dental (veces/semana) } \\
\hline$<7$ & 1.332 & 43,7 \\
\hline$\geq 7$ & 1.716 & 56,3 \\
\hline \multicolumn{3}{|c|}{ Inicio del cepillado dental (años de edad) } \\
\hline$>2$ & 2.361 & 77,7 \\
\hline$\leq 2$ & 679 & 22,3 \\
\hline \multicolumn{3}{|c|}{ Tamaño de la familia (hermanos) } \\
\hline $0-1$ & 1.173 & 38,5 \\
\hline 2 y más & 1.873 & 61,5 \\
\hline \multicolumn{3}{|l|}{ Tipo de escuela } \\
\hline Pública & 2.576 & 84,5 \\
\hline Particular & 472 & 15,5 \\
\hline \multicolumn{3}{|c|}{ Tipo de seguro de salud } \\
\hline Seguridad social & 2.027 & 66,6 \\
\hline Sin seguro & 511 & 16,8 \\
\hline Particular & 506 & 16,6 \\
\hline \multicolumn{3}{|l|}{ Automóvil en el hogar } \\
\hline No & 1.403 & 46,1 \\
\hline Sí & 1.640 & 53,9 \\
\hline \multicolumn{3}{|l|}{ Nivel socioeconómico } \\
\hline 1.er cuartil (peor) & 771 & 25,4 \\
\hline 2.0 & 750 & 24,7 \\
\hline 3.0 & 757 & 25,9 \\
\hline 4. 0 cuartil (mejor) & 759 & 25,0 \\
\hline \multicolumn{3}{|c|}{ Necesidades de salud bucal } \\
\hline Bajas & 543 & 17,8 \\
\hline Moderadas & 773 & 25,4 \\
\hline Altas & 854 & 28,0 \\
\hline Muy altas & 878 & 28,8 \\
\hline
\end{tabular}

servicios preventivos. Entre los niños que utilizaron algún servicio, en promedio se realizaron $1,99 \pm 1,66$ visitas, siendo el promedio más alto para las curativas $(2,01 \pm 1,67)$ que para las preventivas $(1,95 \pm 1,64)$.

\section{Resultados bivariados}

La Tabla 2 muestra la distribución de las prevalencias, mientras que la Tabla 3 muestra la fuerza de la asociación obtenida con regresión logística politómica. Según el tipo de servicio utilizado, las mujeres utilizaron con mayor frecuencia ambos tipos de servicio odontológicos $(p<0,05)$; no obstante, en el análisis de la regresión logística politómica no fue significativa esta diferencia. En cuanto al cepillado dental, los que se cepillaron más de siete veces/semana y los que iniciaron esta costumbre antes de los dos años de edad utilizaron más servicios dentales; el efecto de estas variables fue mayor para los servicios preventivos (Tabla 3). Con relación a los indicadores de posición socioeconómica, la Tabla 2 muestra que en todas las variables existieron diferencias 
Prevalencia de utilización de servicios preventivos y curativos por características seleccionadas de la muestra.

\begin{tabular}{|c|c|c|c|}
\hline Variables & No uso & Curativos & Preventivos \\
\hline \multicolumn{4}{|l|}{ Sexo } \\
\hline Niños & 36,6 & 43,4 & 19,9 \\
\hline Niñas & 32,2 & 46,8 & 20,96 \\
\hline \multicolumn{4}{|c|}{ Frecuencia de cepillado dental (veces/semana) } \\
\hline$<7$ & 41,4 & 42,6 & 16,0 \\
\hline$\geq 7$ & 28,8 & 47,2 & 23,9 \\
\hline \multicolumn{4}{|c|}{ Inicio del cepillado dental (años de edad) } \\
\hline$>2$ & 36,6 & 44,7 & 18,7 \\
\hline$\leq 2$ & 26,2 & 47,2 & 26,6 \\
\hline \multicolumn{4}{|c|}{ Tamaño de la familia (hermanos) } \\
\hline $0-1$ & 31,5 & 46,1 & 22,4 \\
\hline 2 y más & 36,1 & 44,6 & 19,3 \\
\hline \multicolumn{4}{|l|}{ Tipo de escuela } \\
\hline Pública & 36,1 & 45,2 & 18,7 \\
\hline Particular & 24,8 & 45,3 & 29,9 \\
\hline \multicolumn{4}{|c|}{ Tipo de seguro de salud } \\
\hline Seguridad social & 32,8 & 45,3 & 22,9 \\
\hline Sin seguro & 47,6 & 39,7 & 12,7 \\
\hline Particular & 26,7 & 50,6 & 22,7 \\
\hline \multicolumn{4}{|l|}{ Automóvil en el hogar } \\
\hline No & 39,2 & 42,7 & 18,1 \\
\hline Sí & 30,1 & 47,3 & 22,5 \\
\hline \multicolumn{4}{|l|}{ Nivel socioeconómico } \\
\hline 1.er cuartil (peor) & 43,1 & 39,7 & 17,2 \\
\hline $2 . \circ$ & 36,5 & 47,9 & 15,6 \\
\hline $3 . \circ$ & 31,3 & 44,1 & 24,6 \\
\hline 4. ${ }^{\circ}$ cuartil (mejor) & 25,2 & 49,6 & 25,2 \\
\hline \multicolumn{4}{|c|}{ Necesidades de salud bucal } \\
\hline Bajas & 41,8 & 33,1 & 25,1 \\
\hline Moderadas & 35,7 & 41,1 & 23,2 \\
\hline Altas & 34,0 & 48,6 & 17,4 \\
\hline Muy altas & 28,8 & 53,0 & 18,3 \\
\hline
\end{tabular}

significativas $(\mathrm{p}<0,05)$ entre los porcentajes de utilización, destacando el hecho de la disminución de los porcentajes de no utilización, conforme aumentaba el nivel socioeconómico - de $43,1 \%$ a $25,2 \%$. Sin embargo, al analizarlas individualmente por tipo de servicios, empleando regresión logística politómica para los servicios curativos, el tamaño de la familia no tuvo ningún efecto ( $p>0,05)$. Finalmente, las necesidades de salud bucal no resultaron ser significativas para los servicios preventivos ( $\mathrm{p}>0,05)$.

\section{Resultados multivariados}

Los datos de la Tabla 4 muestran las asociaciones ajustadas en regresión logística politómica.
El efecto de la edad fue similar para ambos tipos de servicios: cuando se incrementa la edad, los momios de utilizar algún tipo de servicios odontológicos también se incrementan. La frecuencia de cepillado dental y la edad de inicio de cepillado dental estuvieron asociadas a la utilización de servicios dentales (tanto preventivos como curativos): a mayor frecuencia y menor edad del inicio del cepillado dental, mayor uso de servicios dentales. Un efecto superior de ambas variables sobre los servicios preventivos fue observado y permaneció aún después de ajustar por variables sociodemográficas y socioeconómicas.

Controlando por las variables sociodemográficas y de necesidad, cuatro variables socioeconómicas fueron retenidas en el modelo multi- 
Análisis bivariado de regresión logística multinomial entre las características de la muestra y la utilización de servicios odontológicos ( 0 = sin visitas, 1 = atención preventiva, 2 = atención curativa) en niños escolares.

\begin{tabular}{|c|c|c|}
\hline Variables & $\begin{array}{c}\text { Servicios curativos } \\
\text { RM (IC95\%) }\end{array}$ & $\begin{array}{c}\text { Servicios preventivos } \\
\text { RM (IC95\%) }\end{array}$ \\
\hline Edad del niño & $1,10(1,06-1,16)$ * & $1,12(1,03-1,22) * \star$ \\
\hline \multicolumn{3}{|l|}{ Sexo } \\
\hline Niños & $1 \star \star \star$ & $1 \star \star \star$ \\
\hline Niñas & $1,23(0,98-1,53) \#$ & $1,20(0,96-1,48) \# \#$ \\
\hline \multicolumn{3}{|c|}{ Frecuencia de cepillado dental (veces/semana) } \\
\hline$<7$ & $1 \star \star \star$ & $1 \star \star \star$ \\
\hline$\geq 7$ & $1,59(1,39-1,82)$ \#\#\# & $2,15(1,71-2,70) \# \# \#$ \\
\hline \multicolumn{3}{|c|}{ Inicio del cepillado dental (años de edad) } \\
\hline$>2$ & $1 * \star \star$ & $1 * \star \star$ \\
\hline$\leq 2$ & $1,47(1,15-1,88)$ * & $1,98(1,41-2,78) \# \# \#$ \\
\hline \multicolumn{3}{|c|}{ Tamaño de la familia (hermanos) } \\
\hline $0-1$ & $1 \star \star \star$ & $1 * \star \star$ \\
\hline 2 y más & $0,85(0,69-1,03) \#$ & $0,75(0,61-0,93)$ * \\
\hline \multicolumn{3}{|l|}{ Tipo de escuela } \\
\hline Pública & $1 * \star \star$ & $1 * \star \star$ \\
\hline Particular & $1,46(1,06-2,01) * \star$ & $2,32(1,64-3,28) \# \# \#$ \\
\hline \multicolumn{3}{|c|}{ Tipo de seguro de salud } \\
\hline Seguridad social & $1,65(1,30-2,11) \# \# \#$ & $2,49(1,87-3,32) \# \# \#$ \\
\hline Sin seguro & $1 \star \star \star$ & $1 \star \star \star$ \\
\hline Particular & $2,27(1,77-2,92) \# \# \#$ & 3,18 (2,03-4,99) \#\#\# \\
\hline \multicolumn{3}{|l|}{ Automóvil en el hogar } \\
\hline No & $1 * \star \star$ & $1 * \star \star$ \\
\hline Sí & $1,44(1,16-1,80)$ * & $1,63(1,17-2,26)$ * \\
\hline \multicolumn{3}{|l|}{ Nivel socioeconómico } \\
\hline 1.er cuartil (peor) & $1 * \star \star$ & $1 * \star \star$ \\
\hline $2 . \circ$ & $1,42(1,04-1,94) * \star$ & $1,07(0,79-1,45) \# \#$ \\
\hline $3 . \circ$ & $1,52(1,17-2,00)$ * & $1,97(1,40-2,79)$ \#\#\# \\
\hline 4.o cuartil (mejor) & $2,14(1,61-2,84) \# \#$ & $2,51(1,83-3,44) \# \# \#$ \\
\hline \multicolumn{3}{|c|}{ Necesidades de salud bucal } \\
\hline Bajas & $1 * \star \star$ & $1 * \star \star$ \\
\hline Moderadas & $1,45(1,18-1,79)$ \#\#\# & $1,08(0,80-1,47) \# \#$ \\
\hline Altas & $1,80(1,46-2,22) \# \# \#$ & $0,85(0,65-1,12) \# \#$ \\
\hline Muy altas & $2,32(1,75-3,08) \# \#$ & $1,06(0,77-1,46) \# \#$ \\
\hline
\end{tabular}

Nota: intervalos de confianza calculados con errores estándar robustos por cluster de escuela.

* $p<0,01$;

** $p<0,05$;

$\star \star \star$ Categoría de referencia;

$\# p<0,10$;

\#\# No significativo;

\#\# $\mathrm{p}<0,001$

variado final. Para los servicios curativos, el tipo de escuela no fue significativo $(p>0,05)$ en el modelo final. Para los servicios preventivos, no fueron significativas la posesión de automóvil y el segundo cuartil (comparado con el primero) del nivel socioeconómico. Así, era más probable que los niños utilizaran servicios odontológicos curativos si tenían acceso a la seguridad social pública $(R M=1,37)$ o seguro privado $(R M=1,78)$, si estaban ubicados en los mejores cuartiles del nivel se (segundo; $\mathrm{RM}=1,47$, tercero; $\mathrm{RM}=1,46 \mathrm{y}$ cuarto; $\mathrm{RM}=1,95$ ), y si su familia poseía un automóvil $(R M=1,25 ; p<0,10)$. Por otro lado, el estar inscrito en una escuela particular incrementó un $59 \%$ los momios de utilizar servicios preventivos (IC95\%; 1,14-2,20). Cuando comparamos frente 
Análisis multivariado de regresión logística multinomial entre las características de la muestra y la utilización de servicios odontológicos ( $0=\sin$ visitas, 1 = atención preventiva, 2 = atención curativa) en niños escolares.

\begin{tabular}{|c|c|c|}
\hline Variables & $\begin{array}{c}\text { Servicios curativos } \\
\text { RM (IC95\%) }\end{array}$ & $\begin{array}{c}\text { Servicios preventivos } \\
\text { RM (IC95\%) }\end{array}$ \\
\hline \multicolumn{3}{|c|}{ Factores predisponentes } \\
\hline Edad del niño & $1,13(1,06-1,19)$ * & $1,14(1,04-1,25)$ ** \\
\hline \multicolumn{3}{|c|}{ Frecuencia de cepillado dental (veces/semana) } \\
\hline$<7$ & $1 \star \star \star$ & $1 \star \star \star$ \\
\hline$\geq 7$ & $1,41(1,23-1,62)$ * & $1,68(1,31-2,17)$ * \\
\hline \multicolumn{3}{|c|}{ Inicio del cepillado dental (años de edad) } \\
\hline$>2$ & $1 * \star \star$ & $1 \star \star \star$ \\
\hline$\leq 2$ & $1,47(1,10-1,95) * \star$ & $1,88(1,30-2,71) * \star$ \\
\hline \multicolumn{3}{|l|}{ Factores facilitantes } \\
\hline \multicolumn{3}{|l|}{ Tipo de escuela } \\
\hline Pública & $1 * \star \star$ & $1 * \star \star$ \\
\hline Particular & $1,04(0,75-1,43) \#$ & $1,59(1,14-2,20)$ ** \\
\hline \multicolumn{3}{|c|}{ Tipo de seguro de salud } \\
\hline Seguridad social & $1,37(1,12-1,68)$ ** & $1,86(1,31-2,64) * *$ \\
\hline Sin seguro & $1 * \star \star$ & $1 * \star \star$ \\
\hline Particular & $1,78(1,46-2,18)$ * & $2,09(1,38-3,16)$ * \\
\hline \multicolumn{3}{|l|}{ Automóvil en el hogar } \\
\hline No & $1 * \star \star$ & $1 * \star \star$ \\
\hline Sí & $1,25(0,99-1,57)$ \#\# & $1,19(0,88-1,62) \#$ \\
\hline \multicolumn{3}{|l|}{ Nivel socioeconómico } \\
\hline 1.er cuartil (peor) & $1 * \star \star$ & $1 \star \star \star$ \\
\hline $2 . \circ$ & $1,47(1,08-2,01) \# \# \#$ & $1,01(0,74-1,36) \#$ \\
\hline $3 . \circ$ & $1,46(1,07-1,98)$ \#\#\# & 1,58 (1,05-2,39) \#\#\# \\
\hline 4. ${ }^{\circ}$ cuartil (mejor) & $1,95(1,43-2,68)$ * & $1,70(1,25-2,30)$ ** \\
\hline \multicolumn{3}{|l|}{ Necesidades } \\
\hline \multicolumn{3}{|c|}{ Necesidades de salud bucal } \\
\hline Bajas & $1 * \star \star$ & $1 \star \star \star$ \\
\hline Moderadas & $1,51(1,22-1,87)$ * & $1,17(0,81-1,70) \#$ \\
\hline Altas & $2,06(1,70-2,50)$ * & $1,05(0,77-1,44) \#$ \\
\hline Muy altas & $2,78(2,12-3,65)$ * & $1,33(0,95-1,85) \#$ \\
\hline
\end{tabular}

Nota: modelo ajustado por las variables contenidas en el cuadro además de sexo; los intervalos de confianza fueron calculados con errores estándar robustos por cluster de escuela.

* $p<0,001$;

** $\mathrm{p}<0,01$;

*** Categoría de referencia;

\# No significativo:

$\#$ \# $<0,10$;

\#\# $\mathrm{p}<0,05$

a los niños sin seguro, los niños con acceso a la seguridad social pública aumentaron la posibilidad de tener alguna visita preventiva $(R M=1,86)$, mientras que los niños con seguro privado lo incrementaron aún más $(\mathrm{RM}=2,09)$. En cuanto al nivel socioeconómico, los niños del tercer y cuarto cuartil (los de mejor posición socioeconómica) utilizaron más los servicios de salud preventivos ( $R M=1,58, R M=1,70$, respectivamente) que los niños de los cuartiles inferiores.
Finalmente, se estimó que los niños que tuvieron mayores necesidades de salud utilizaron más los servicios curativos: los momios de utilizar servicios curativos en los niños de necesidades bucales moderadas, altas y muy altas incrementaron 1,51 (IC95\%: 1,22-1,87), 2,06 (IC95\%: 1,70-2,50), y 2,78 (IC95\%: 2,12-3,65) veces, comparado con los momios de utilización de los niños de necesidades bajas. Para la utilización de servicios preventivos, las necesidades de salud 
bucal no tuvieron efecto alguno ( $\mathrm{p}>0,05)$ cuando este modelo incluyó las demás variables independientes.

\section{Discusión}

Comúnmente, los estudios sobre utilización de servicios de salud odontológicos que se han realizado alrededor del mundo se llevan a cabo en población adulta y en países industrializados. El presente trabajo representa un esfuerzo por incrementar la información disponible sobre ese tipo de servicios de salud en niños de un país en desarrollo. Los principales hallazgos indican que diversas variables de posición socioeconómica estuvieron asociadas a la utilización de servicios de salud bucal. La asociación observada fue: cuanto mejor posición socioeconómica se observaba, la utilización fue mayor; esto sugiere la existencia de desigualdades socioeconómicas en la utilización de servicios de salud bucal. Estas desigualdades han sido documentadas en el perfil epidemiológico de la población infantil urbana $14,18,19$, adulta e infantil peri-urbana marginal 13,20, pero no necesariamente entre la urbana y la remota rural en los grupos infantil y de la tercera edad 50,51.

En México, que tiene un sistema de salud fragmentado en varios sub-sistemas, los servicios de salud bucodental se ofrecen a través de varias organizaciones aisladas. La primera es el Ministerio o Secretaría de Salud, que ofrece servicios para la población no derechohabiente. La Seguridad Social ofrece servicios para los trabajadores de los sectores público y estatal, y son proporcionados por el Instituto Mexicano del Seguro Social (IMSS), el Instituto de Seguridad y Servicios Sociales para los Trabajadores del Estado (ISSSTE), el Instituto de Seguridad Social para las Fuerzas Armadas Mexicanas (ISSFAM), y por Petróleos Mexicanos (PEMEX). Por último, los servicios privados que son pagados por las empresas o personas que los demandan. Desafortunadamente, los servicios públicos sólo cubren una pequeña porción de los tratamientos que requiere la población. Las actividades de servicio incluyen: (a) obturaciones; (b) terapias pulpares; (c) cirugías bucales, y (d) extracciones de piezas dentales. En materia de prevención, y en el marco de la salud bucal del preescolar y escolar, se realizan una serie de acciones preventivas que incluyen enjuagues bucales y/o aplicaciones tópicas de fluoruros, instrucciones de la técnica de cepillado, acciones de control de placa dentobacteriana, e instrucciones en el uso del hilo dental. Aunque en años recientes se ha tratado de incrementar el acceso a los servicios de salud públicos, los de sa- lud bucal no han estado incluidos en este rubro, por lo el grueso de la población normalmente accede a estos servicios realizando un buen porcentaje del gasto privado en salud 52: a través de ser ofertados en los servicios de salud privados, ofrecen una amplia gama de tratamiento, pero con el riesgo de caer en gasto catastrófico en la población de menor posición socioeconómica. Aunado a esto, estudios recientes demuestran que este tipo de servicio tiene una baja cobertura efectiva en la población mexicana 26 .

En el mismo sentido que han reportado otros autores, en el presente estudio se observaron que variables como aseguramiento, nivel socioeconómico, educación, y ocupación son predictores de la utilización de servicios de salud odontológi$\cos 22,23,24,31,32,33,34,35,36$. El mecanismo que tiene la posición socioeconómica sobre la salud no es claro aún 53 , pero con respecto a los servicios de salud bucal podría especularse sobre los distintos mecanismos. Por un lado, al no ser provistos por el subsistema de salud público, los precios en el sector privado pueden ser más altos de los que la población está dispuesta a pagar, constituyendo de esta manera una importante barrera económica de acceso. Por otro, están las percepciones que tienen las personas hacia la salud bucal. Diversos autores han mencionado que para muchas personas (y se podría pensar que también para algunos sistemas de salud) la salud bucal no forma parte de la salud integral; se la ve como una cuestión/tema aparte, ya que la mayor parte de las veces los problemas dentales no causan muertes en la población 13,26. Alentadoramente, ha habido recientemente en México posiciones oficiales que hacen mención sobre la importancia que tiene la salud bucal en la población 54 .

Fue interesante encontrar mediante nuestros análisis que - al igual que en otros estudios - el modelo de regresión mostró que las variables sociodemográficas como edad 22,23,24,34 y las actitudes y conductas - como el cepillado dental 22,23,24 - tuvieron una asociación con la utilización de servicios de salud bucal, independientemente de la posición socioeconómica y de las necesidades de salud.

Los resultados del estudio permiten afirmar que las necesidades de salud son importantes variables en la predicción de la utilización de servicios de salud bucal. La mayor importancia del estudio reside en que se pudo discriminar el impacto por tipo de servicios y se utilizó como variable proxy de necesidad de salud la experiencia de caries: aún después de ajustar por variables sociodemográficas, se observó una interesante asociación en la que los individuos que presentaron mayores necesidades, utilizaron más servicios de salud curativos. Sin embargo, esta relación no fue 
significativa para los servicios preventivos. Como ha sido confirmado en varios estudios, tanto en niños como en adultos, empleando diferentes indicadores de necesidades de salud bucal como mayor cantidad de dientes naturales presentes, la presencia de dentaduras, o incluso la autopercepción de necesidades 22,23,24,36, los que presentan mayor necesidad utilizan más los servicios de salud bucal.

Existen dos limitaciones principales que sugieren precaución al hacer conclusiones sobre los hallazgos del presente estudio. La relación causal observada entre las variables independientes y la utilización de servicios no puede ser establecida debido a la naturaleza transversal de los datos. Los efectos son limitados al área urbana y no pueden ser generalizados a pueblos pequeños, áreas rurales, o suburbanas. A modo de conclusión general, podemos decir que los resultados permiten sugerir la existencia de desigualdades socioeconómicas en la utilización de servicios de salud bucal en niños mexicanos, tanto en servicios curativos, como preventivos. Esta población en el noroeste de México, es una región en la cual no se han llevado a cabo evaluaciones epidemiológicas o de servicios de salud de gran alcance. Esto pone de manifiesto la necesidad de incrementar el esfuerzo para combatir las desigualdades en salud en México, específicamente en este tipo de entorno urbano menor, en el noroeste del país. Los estudios subsecuentes deben enfocarse a establecer qué tan adecuada es la utilización de servicios bucales, esto es, si el tipo de servicio utilizado es el apropiado para satisfacer las necesidades reales del individuo - de acuerdo a criterios normativos, o de necesidad sentida o expresada.

\section{Resumen}

El objetivo del estudio fue evaluar la asociación entre diversos indicadores socioeconómicos y la utilización de servicios de salud bucal. Se realizó un estudio transversal en 3.048 escolares. La variable utilización de servicios de salud bucal y las variables sociodemográficas y socioeconómicas se colectaron a través de un cuestionario dirigido a las madres. Para determinar las necesidades de salud se realizó un examen clínico bucal a los niños. Las asociaciones ajustadas fueron evaluadas con regresión logística politómica. Las variables asociadas a servicios preventivos y curativos fueron mayor edad, mayor frecuencia de cepillado y menor edad al inicio de cepillado dental, tener seguro público o privado, y mejor nivel socioeconómico. Además, sólo para los servicios preventivos, también se asoció estar inscrito en una escuela privada, y sólo para los servicios curativos, la posesión de automóvil y tener necesidades de salud bucal entre moderadas $y$ muy altas. Los resultados sugieren la existencia de desigualdades socioeconómicas en la utilización de servicios de salud bucal en niños mexicanos.

Servicios de Salud Dental; Investigación sobre Servicios de Salud; Desigualdades en Salud; Niño

\section{Colaboradores}

C. E. Medina-Solís y J. J. Villalobos-Rodelo concibieron el estudio, supervisaron todos los aspectos en la realización del mismo y realizaron los análisis estadísticos. M. L. Márquez-Corona, A. A. Vallejos-Sánchez, C. López Portillo-Núñez y A. J. Casanova-Rosado contribuyeron al análisis e interpretación de los datos y a la redacción del manuscrito. Todos los autores aportaron ideas, interpretaron los hallazgos y revisaron los borradores del manuscrito. Todos los autores aprobaron la versión final. C. E. Medina-Solís es el responsable del artículo. 


\section{Referencias}

1. Bradley CJ, Given CW, Roberts C. Health care disparities and cervical cancer. Am J Public Health 2004; 94:2098-103.

2. Ávila-Burgos L, Ramírez-Valverde G, MartínezDamián MA, Cruz-Valdez A, Santiago-Cruz MJ, Medina-Solís CE. Socio-economic determinants of inequality and self-reported morbidity among adolescents in a developing country. Saudi Med J 2005; 26:1617-23.

3. Chen E, Martin AD, Matthews KA. Understanding health disparities: the role of race and socioeconomic status in children's health. Am J Public Health 2006; 96:702-8.

4. James SA, Fowler-Brown A, Raghunathan TE, Van Hoewyk J. Life-course socioeconomic position and obesity in African American women: the Pitt County Study. Am J Public Health 2006; 96:554-60.

5. James SA, Van Hoewyk J, Belli RF, Strogatz DS, Williams DR, Raghunathan TE. Life-course socioeconomic position and hypertension in African American men: the Pitt County Study. Am J Public Health 2006; 95:812-7.

6. Rosvall M, Chaix B, Lynch J, Lindstrom M, Merlo J. Contribution of main causes of death to social inequalities in mortality in the whole population of Scania, Sweden. BMC Public Health 2006; 6:79.

7. Morales LS, Staiger D, Horbar JD, Carpenter J, Kenny M, Geppert J, et al. Mortality among very lowbirthweight infants in hospitals serving minority populations. Am J Public Health 2005; 95:2206-12.

8. Esnaola S, Aldasoro E, Ruiz R, Audicana C, Pérez Y, Calvo M. Desigualdades socioeconómicas en la mortalidad en la Comunidad Autónoma del País Vasco. Gac Sanit 2006; 20:16-24.

9. Petrelli A, Gnavi R, Marinacci C, Costa G. Socioeconomic inequalities in coronary heart disease in Italy: a multilevel population-based study. Soc Sci Med 2006; 62:446-56.

10. Braveman PA, Egerter SA, Cubbin C, Marchi KS. An approach to studying social disparities in health and health care. Am J Public Health 2004; 94:2139-48.

11. Cano-Serral G, Rodríguez-Sanz M, Borrell C, Pérez MM, Salvador J. Desigualdades socioeconómicas relacionadas con el cuidado y el control del embarazo Gac Sanit 2006; 20:25-30.

12. Ash M, Brandt S. Disparities in asthma hospitalization in Massachusetts. Am J Public Health 2006; 96:358-62.

13. Maupomé G. An introspective qualitative report on dietary patterns and elevated levels of dental decay in a deprived urban population in northern Mexico. ASDC J Dent Child 1998; 65:276-85.

14. Irigoyen ME, Maupomé G, Mejía AM. Caries experience and treatment needs in a 6- to 12-year-old urban population in relation to socio-economic status. Community Dent Health 1999; 16:245-9.

15. Nicolau B, Marcenes W, Bartley M, Sheiham A. Associations between socio-economic circumstances at two stages of life and adolescents' oral health status. J Public Health Dent 2005; 65:14-20.
16. Peres MA, Peres KG, Traebert J, Zabot NE, Lacerda JT. Prevalence and severity of dental caries are associated with the worst socioeconomic conditions: a Brazilian cross-sectional study among 18-yearold males. J Adolesc Health 2005; 37:103-9.

17. Fernandes LS, Peres MA. Associação entre atenção básica em saúde bucal e indicadores socioeconômicos municipais. Rev Saúde Pública 2005; 39:930-6.

18. Casanova-Rosado AJ, Medina-Solís CE, Casanova-Rosado JF, Vallejos-Sánchez AA, Maupomé G, Ávila-Burgos L. Dental caries and associated factor in Mexican schoolchildren aged 6-13 years. Acta Odontol Scand 2005; 63:245-51.

19. Segovia-Villanueva A, Estrella-Rodríguez R, Medina-Solís CE, Maupomé G. Dental caries experience and factors among preschoolers in Southeastern Mexico: a brief communication. J Public Health Dent 2006; 66:88-91.

20. Medina-Solis CE, Pérez-Núñez R, Maupomé G, Casanova-Rosado JF. Edentulism among Mexicans 35 years old and older, and associated factors. Am J Public Health 2006; 96:1578-81.

21. Antunes JL, Peres MA, Mello TR, Waldman EA. Multilevel assessment of determinants of denta caries experience in Brazil. Community Dent Oral Epidemiol 2006; 34:146-52.

22. Medina-Solís CE, Casanova-Rosado AJ, CasanovaRosado JF, Vallejos-Sánchez AA, Maupomé G, Ávila-Burgos L. Factores socioeconómicos y dentales asociados a la utilización de servicios dentales en escolares de Campeche, México. Bol Med Hosp Infant Mex 2004; 61:324-33.

23. Medina-Solís CE, Maupomé G, Ávila-Burgos L, Casanova-Rosado JF, Vallejos-Sánchez AA, Segovia-Villanueva A. Utilización de servicios odontológicos de salud por niños menores de 5 años con seguridad social. Rev Mex Pediatr 2004; 71:222-8.

24. Medina-Solís CE, Maupomé G, Ávila-Burgos L, Hijar-Medina M, Segovia-Villanueva A, Pérez-Núñez R. Factors influencing the use of dental health services by preschool children in Mexico. Pediatr Dent 2006; 28:285-92.

25. Sánchez-García S, De La Fuente-Hernández J, Juárez-Cedillo T, Mendoza JM, Reyes-Morales H, Solórzano-Santos F, et al. Oral health service utilization by elderly beneficiaries of the Mexican Institute of Social Security in México city. BMC Health Serv Res 2007; 7:211.

26. Pérez-Núñez R, Medina-Solis CE, Maupomé G, Vargas-Palacios A. Factors associated with dental health care coverage in Mexico: findings from the National Performance Evaluation Survey 20022003. Community Dent Oral Epidemiol 2006; 34:387-97.

27. Thomson WM. Use of dental services by 26 -yearold New Zealanders. N Z Dental J 2001; 97:44-8.

28. Petersen PE, Pedersen KM. Socioeconomic demand model for dental visits. Community Dent Oral Epidemiol 1984; 12:361-5. 
29. Pavi E, Kay EJ, Stephen KW. The effect of social and personal factors on utilization of dental services in Glasgow, Scotland. Community Dent Health 1995; 12:208-15.

30. Manski RJ, Magder LS. Demographic and socioeconomic predictors of dental care utilization. J Am Dent Assoc 1998; 128:195-200.

31. Kiyak HA. An explanatory model of older persons' use of dental services. Implications for health policy. Med Care 1987; 25:936-52.

32. Waldman HB. Dental insurance coverage and the use of dental services by children. ASDC J Dent Child 1989; 56:125-8.

33. Adegbembo AO. Household utilization of dental services in Ibadan, Nigeria. Community Dent Oral Epidemiol 1994; 22:338-9.

34. McCormick MC, Kass B, Elixhauser A, Thompson J, Simpson L. Annual report on access to and utilization of health care for children and youth in the United States-1999. Pediatrics 2000; 105:219-30.

35. Lo EC, Schwarz E. Determinants for dental visit behavior among Hong Kong Chinese in a longitudinal study. J Public Health Dent 1998; 58:220-7.

36. Stewart DCL, Ortega AN, Dausey D, Rosenheck R. Oral health and use of dental services among Hispanics. J Public Health Dent 2002; 62:84-91.

37. Grembowski D, Andersen RM, Chen MS. A public health model of the dental care process. Med Care Rev 1989; 46:439-96.

38. Andersen RM, Davidson PL. Improving access to care in America: individual and contextual indicators. In: Andersen RM, Rice TH, Kominski GF, editors. Changing the U.S. health care system: key issues in health services policy and management. 3rd Ed. San Francisco: Jossey-Bass; 2007. p. 3-31.

39. Davidson OL, Andersen RM. Determinants of dental care utilization for diverse ethnic and age group. Adv Dent Res 1997; 11:254-62.

40. Matos DL, Lima-Costa MFF, Guerra HL, Marcenes W. Projeto Bambuí: estudo de base populacional dos fatores associados com o uso regular de serviços odontológicos em adultos. Cad Saúde Pública 2001; 17:661-8.

41. Scheutz F, Heidmann J. Determinants of utilization of dental services among 20- to 34-year-old Danes. Acta Odontol Scand 2001; 59:201-8.

42. Villalobos-Rodelo JJ, Medina-Solís CE, Maupomé G, Vallejos-Sánchez AA, Lau-Rojo L, De León-Viedas MV. Socioeconomic and sociodemographic variables associated with oral hygiene status in Mexican schoolchildren aged 6 to 12 years. J Periodontol 2007; 78:816-22.
43. Villalobos-Rodelo JJ, Medina-Solís CE, Maupomé G, Pontigo-Loyola AP, Lau-Rojo L, Verdugo-Barraza L. Caries dental en escolares de una comunidad del Noroeste de México con dentición mixta, y su asociación con algunas variables clínicas, socioeconómicas y sociodemográficas. Rev Invest Clin 2007; 59:256-67.

44. World Health Organization. Oral health survey - basics methods. $4^{\text {th }}$ Ed. Geneva:World Health Organization; 1997.

45. Pitts NB. Clinical diagnosis of dental caries: a European perspective. J Dent Educ 2001; 65:972-8.

46. Kolenikov S, Angeles G. The use of discrete data in PCA: theory, simulations, and applications to socioeconomic indices. Chapel Hill: Carolina Population Center, University of North Carolina; 2004. (CPC/MEASURE Working Paper, WP-04-85).

47. Hosmer D, Lemeshow S. Applied logistic regression. $2^{\text {nd }}$ Ed. New York: Wiley-Interscience Publication; 2000

48. Bagley SC, White H, Golomb BA. Logistic regression in the medical literature: Standards for use and reporting, with particular attention to one medical domain. J Clin Epidemiol 2001; 54:979-85.

49. Williams RL. A note on robust variance estimation for cluster-correlated data. Biometrics 2000; 56:645-6.

50. Borges-Yáñez SA, Maupomé G, Martínez-González M, Cervantez-Turrubiante L, Gutiérrez-Robledo LM. Dietary fiber intake and dental health status in urban-marginal, and rural communities in central Mexico. J Nutr Health Aging 2004; 8:333-9.

51. Irigoyen ME, Luengas IF, Yashine A, Mejía AM, Maupomé G. Dental caries experience in Mexican schoolchildren from rural and urban communities. Int Dent J 2000; 50:41-5.

52. Pérez-Núñez R, Vargas-Palacios A, Ochoa-Moreno I, Medina-Solis CE. Household expenditure in dental health care: national estimations in Mexico for 2000, 2002, and 2004. J Public Health Dent 2007; 67:234-42.

53. Laaksonen M, Rahkonen O, Martikainen P, Lahelma E. Socioeconomic position and self-rated health: the contribution of childhood socioeconomic circumstances, adult socioeconomic status, and material resources. Am J Public Health 2005; 95:1403-9.

54. Frenk J. Estimados cirujanos dentistas. Rev Mex Odon Clín 2006; 1:2.

Recibido el 06/May/2009

Versión final presentada el 28/Ago/2009

Aprobado el 30/Sep/2009 